\title{
Preface to the special issue dedicated to the 2015 World Congress on Global Optimization
}

\author{
Panos M. Pardalos ${ }^{1}$
}

Received: 5 April 2017 / Accepted: 7 April 2017 / Published online: 11 May 2017

(C) Springer-Verlag Berlin Heidelberg 2017

During February 22-15, 2015, the 2015 World Congress on Global Optimization (WCGO IV) took place at the University of Florida. The congress was organized by the International Society of Global Optimization, the Center for Applied Optimization, and the Department of Industrial at the University of Florida, Gainesville, FL, USA.

The participants came from all over the world and the talks covered a wide spectrum of topics of global optimization including new theoretical results, algorithmic and software developments, and applications.

This special issue of Optimization Letters contains selected refereed papers presented at the 2015 World Congress on Global Optimization. The papers focus on theoretical and computational aspects of global optimization.

I would like to take this opportunity to thank all contributors and reviewers for their excellent work during the editing process of this special issue. The collection

of papers in this issue reflects the interdisciplinary nature of global optimization and recent directions of research.

Panos M. Pardalos

pardalos@ufl.edu

1 Center for Applied Optimization, University of Florida, Gainesville, FL, USA 